\title{
Removal of extra sequences with I-Scel in combination with CRISPR/Cas9 technique for precise gene editing in Drosophila
}

\author{
Nikolay Zolotarev', Pavel Georgiev² \& Oksana Maksimenko*,1
}

\section{ABSTRACT}

The CRISPR/Cas9 system has recently emerged as a powerful tool for functional genomic studies and has been adopted for many organisms, including Drosophila. Previously, an efficient two-step strategy was developed to engineer the fly genome by combining CRISPR/Cas9 with recombinase-mediated cassette exchange (RMCE). This strategy allows the introduction of designed mutations into a gene of interest in vivo. However, the loxP or frt site remains in the edited locus. Here, we propose a modification of this approach for rapid and efficient seamless genome editing with CRISPR/Cas9 and site-specific recombinase-mediated integration (SSRMI) combined with recombination between homologous sequences induced by the rare-cutting endonuclease I-Scel. The induced homological recombination leads to the removal of the remaining extraneous sequences from the target locus.

\section{METHOD SUMMARY}

Modification of the method for Drosophila genome editing that allows different mutations to be easily and precisely introduced into a gene through three subsequent steps, namely, CRISPR/Cas9, SSRMI and I-Scel-mediated recombination techniques.

\section{KEYWORDS:}

CRISPR/Cas9 - genome editing - homologous recombination

'Group of Molecular Organization of Genome, Russian Academy of Sciences, 34/5 Vavilov St, Moscow 119334, Russia; ${ }^{2}$ Department of the Control of Genetic Processes, Institute of Gene Biology, Russian Academy of Sciences, 34/5 Vavilov St, Moscow 119334, Russia; *Author for correspondence: maksog@ mail.ru

BioTechniques 66: 198-201 (April 2019) 10.2144/ btn-2018-0147
Genome editing is a powerful tool for dissecting biological mechanisms of a particular gene. Opportunities for precisely introducing designed mutations into a gene have expanded significantly in recent years with the development of a simple CRISPR/ Cas 9 system for introducing double-strand breaks into the designed sequences [1,2]. In Drosophila, the efficient method of CRISPR/ Cas9-mediated homology-directed repair (HDR) has been developed $[3,4]$, in which double-stranded DNA (dsDNA) donors are used to facilitate substitution of the targeted gene by exogenous sequences, including easily screened and removable visible markers. The combination of this approach with recombinase-mediated cassette exchange (RMCE) [5] provides a simple and effective two-step method for introducing any changes into a gene of interest [6]. A limitation of this method is that loxP and $a t t R$ sites are retained in the gene after the introduction of the designed sequences $[3,6,7]$. These extra sequences may interfere with proper function of regulatory sequences located in the $5^{\prime}$ region of a target gene or a neighboring gene. This is a common problem in Drosophila, since approximately $31.6 \%$ of all genes are located in the head-to-head orientation, and frequently (59.0\%) promoters of such genes are closely spaced, and transcription start sites (TSSs) are located at a distance of less than $400 \mathrm{bp}$ [8].

To overcome possible problems with the presence of additional sequences in the edited gene, we have introduced I-Scel sites into the landing platform sequence and the replacement construct (Figure 1). The I-Scel endonuclease is encoded by an intron of the large mitochondrial rRNA in yeast [9]. As shown in biochemical studies, I-Scel has an 18-bp specificity and leaves a 4-bp $5^{\prime}$ overhang after the cleavage [10]. This endonuclease is widely used to induce DNA double-strand breaks (DSBs) at I-Scel target sites in the Drosophila genome [11,12]. In particular, an I-Scel endonuclease-based method was used to efficiently delete sequences located between direct repeats with a length of only 126 nucleotides [13]. In the replacement construct, DSBs generated by I-Scel induce recombination between homologous sequences that results in the removal of all extra sequences from the edited gene (Figure 1).

We used the CG6808 gene to test this variant of the editing approach (Figure 2A). There are two isoforms of the $C G 6808$ gene with the same coding sequence. The large isoform overlaps with the CG14711 gene, whose TSS is close to that of the short CG6808 isoform. In this situation, it was difficult to delete CG6808 without affecting CG14711. The gRNAs for Cas9-mediated deletion of CG6808 (approximately $1.5 \mathrm{~kb}$ ) and the sequences for homologous recombination were chosen as shown in Figure 2A.

We injected a mixture of gRNA coding plasmid and homologous recombination plasmid (1:10) into Drosophila embryos (line \#58492 from the Bloomington Drosophila Stock Center). A total of 200 embryos were injected, and flies with gene deletion were selected based on expression of the mCherrygene under control of Actin $5 \mathrm{C}$ promoter. Five different lines were obtained from two independent crossings. The results of introducing the landing platform were checked by PCR (Figure 2B), and the insertion size was found to be correct in all the lines. We also obtained viable homozygous flies, which indicated that the CG6808 deletion did not affect fly viability and that there also was no lethal off-target effect.

Homozygous flies were crossed with the line expressing $\varphi C 37$ integrase under control of vasa promoter [14]. The resulting line carrying the landing platform and 


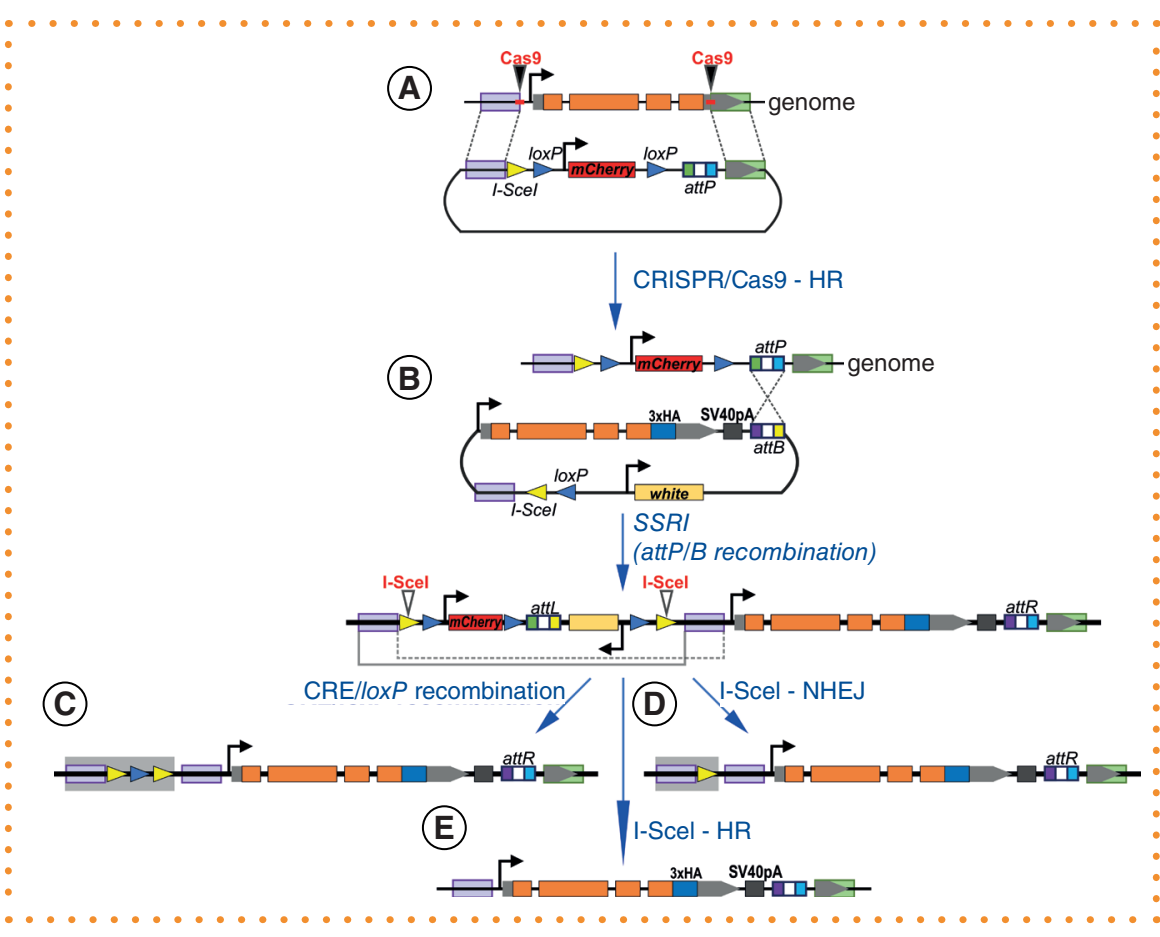

Figure 1. Scheme of gene editing using I-Scel endonuclease in combination with CRISPR/Cas9 and site-specific recombinase-mediated integration (SSRMI) techniques. (A) Scheme of the genome engineering on the model of CG6808 gene using CRISPR/Cas9 combined with homologous recombination (HR). (B) Scheme of SSRMI of the construct containing the edited gene. (C) Result of CRE/ lox $P$ recombination aimed to delete exogenous sequences from the genome. (D) Result of I-Scel restriction with subsequent ligation of the restricted ends (NHEJ). (E) Result of I-Scel restriction with subsequent $\mathrm{HR}$ that allow a recovery of the native genomic background. HR: Homologous recombination; NHEJ: Nonhomologous end joining.

- expressing $\varphi$ C31 integrase was used for injections of the rescue plasmid, which could also be co-injected with the integrase-coding plasmid. It was found that attP/B recombination was much more effective than CRISPR/Cas9-homologous recombination (HR). In total, 100 embryos were injected, and ten independent lines with the insertion were selected based on the white reporter gene expression.

Males with the rescue construct were individually crossed with females carrying the I-Scel gene under control of hsp70 promoter (line \#6934 from the Bloomington Drosophila Stock Center). Embryos were heat-shocked three times for $3 \mathrm{~h}$ at $37^{\circ} \mathrm{C}$ starting 2 days after egg laying to induce I-Scel expression. Flies with successful cutting-out were selected based on changes in the white and mCherry phenotype. All adult flies had a mosaic white phenotype (Figure 2C), indicating the efficiency of I-Scel-mediated deletions. The proportion of flies with I-Scel cutting-out (mosaic white phenotype) ranged from 66 to $88 \%$ in different crossings. In the next generation, we obtained approximately $86 \%$ transgenic flies that lost the white and mcherry phenotype. For further study, we selected independent transgenic males (white eyes and no mCherry expression) and individually crossed them with TM6,Hu/MKRS females in order to obtain homozygous lines.

The DSBS induced by I-Scel can be repaired by $\mathrm{HR}$ or nonhomologous end joining (NHEJ). HR provides for a seamless junction between the genomic sequence and the rescue construct, while NHEJ leads to ligation of the cutting ends and duplication of homologous sequences at the junction (see Figure 1D,E). To test these possibilities against each other, we performed PCR with primers flanking the edited region (Figure 2D). The PCR products from all eight of the tested lines had the same size as the wild-type, confirming the extremely high efficiency of the seamless homologous recombination (Figure 2D). We sequenced these PCR products and confirmed that they were the same as the wild-type sequence. To verify the absence of contamination by wild-type flies, we performed qPCR for hemagglutinin $(3 \times \mathrm{HA})$ sequence fused with the 3'part of the edited CG6808 coding region (Figure $2 \mathrm{E}$ ). As a result, we confirmed that both alleles contained $3 \times \mathrm{HA}$ (i.e., both alleles were with the replacement construct). We also demonstrated an extremely high frequency of not only cutting out all the additional construct sequences flanked by I-Scel (about 70\%), but also of completely restoring the genetic environment by homologous sequences ( $100 \%$ of amplified and sequenced cases). Thus, our results provide evidence that the modified strategy combining CRISPR/Cas9, SSRMI, and I-Scelmediated recombination allows efficient gene editing with precise restoration of 5 '-regulatory regions in vivo.

The approach described here allows the introduction of any mutations in the sequence of the target gene without affecting the 5 '-regulatory region of this gene, as well as an adjacent gene (Figure $3 \mathrm{~A}$ ). Importantly, it is possible to select the best variant of a guide RNA in the extended genomic region limited only on one side by the coding region of a gene of interest. At the same time, this approach has limitations. Additional sequences that remain in the $3^{\prime}$ regions of the gene after genomic manipulation might in some cases affect proper maturation of mRNAs or affect expression of a neighboring gene. Depending on the specific organization of genes in the edited genomic region, it would be possible either to duplicate the critical $3^{\prime}$ region or to use, in addition to the $\mathrm{I}-\mathrm{Scel}$, another endonuclease like I-CREI $[15,16]$ with similar properties for seamless editing at the 3 ' region (Figure $3 \mathrm{~B}$ ). Also, in some cases the approach that combines CRISPR/Cas9 with SSRMI has limitations when deleting sequences larger than $3 \mathrm{~kb}$. In such cases, it would be possible to generate smaller deletions leaving a duplication of the 3 ' region of a target gene (Figure 3C).

\section{AUTHOR CONTRIBUTIONS}

$P G$ and $O M$ conceived the study. NZ performed experimental work. All authors analyzed data. OM provided supervision. PG and $\mathrm{OM}$ wrote the manuscript. OM provided funding.

\section{ACKNOWLEDGMENTS}

We thank $F$ Hasanov for fly injections and NA Gorgolyuk and V Mogila for help in preparing the manuscript. 
(A)

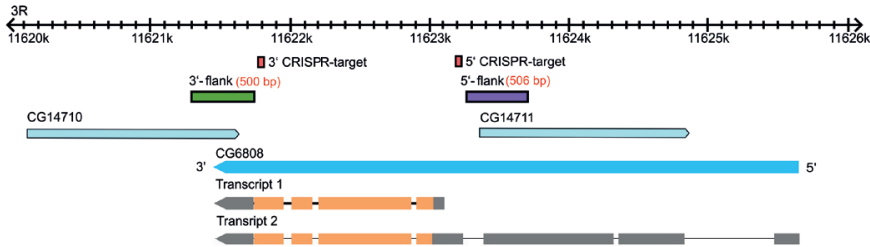

(B) $\mathrm{M}$
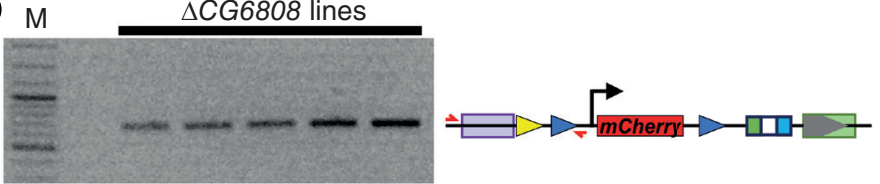

M wt $\Delta C G 6808$ lines

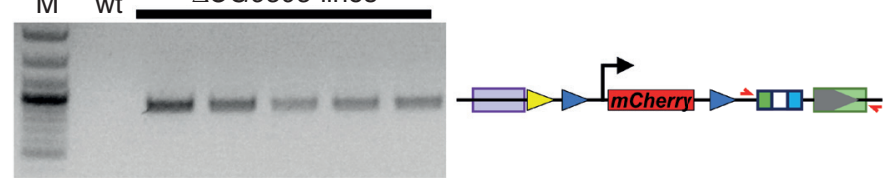

(C)
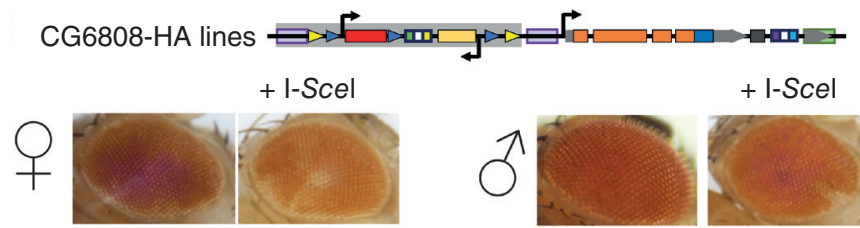

+ I-Scel

(D)

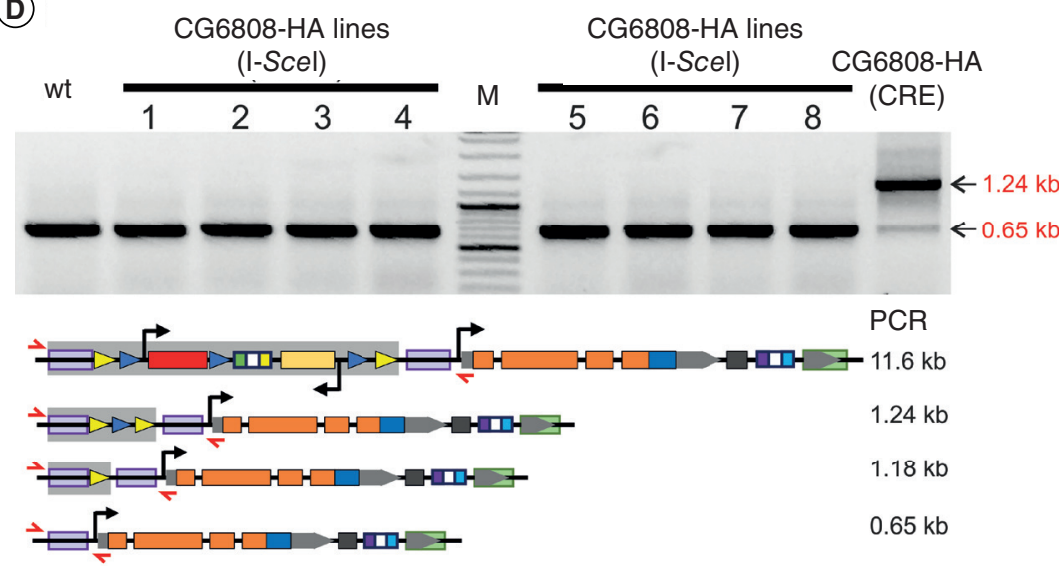

(E)

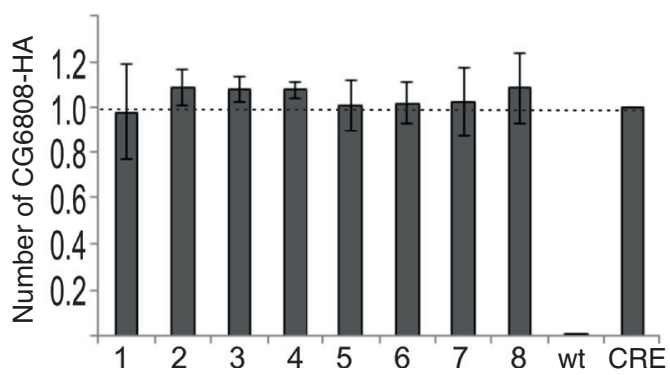

\section{FINANCIAL \& COMPETING INTERESTS DISCLOSURE}

This study was supported by the Russian Foundation of Basic Researches, project no. 18-29-07081 (to OM). This study was performed using the equipment of the
IGB RAS Core Facilities Centre, supported by the Ministry of Science and Education of the Russian Federation. The authors have no other relevant affiliations or financial involvement with any organization or entity with a financial
Figure 2. Results of CG6808 editing. (A) Scheme of the $C G 6808$ gene in the genomic context. Red boxes represent the chosen CRISPR targets; violet and green boxes, flanking homologous regions used in the construct for homologous recombination. (B) Detection of lines with the deleted CG6808 gene after CRISPR/Cas9 editing with subsequent homologous recombination. Gel electrophoresis images show PCR products of $5^{\prime}$ - and 3 '-edited regions of $C G 6808$ (corresponding primers are shown as red arrowheads in schemes on the right). (C) The eyes of female and male flies from the lines obtained after attP/attB recombination and the mosaic eyes of flies from the lines obtained after CRE/ loxP recombination. (D) PCR testing of fly lines after I-Scel restriction. The schemes below show all potential variants with estimated sizes of PCR products: the initial lines and the lines obtained after $\mathrm{CRE} /$ /ox $P$ recombination, after I-Scel restriction with subsequent ligation of the restricted ends, and after I-Scel restriction with subsequent homologous recombination. 1,...,8: Different fly lines after I-Scel restriction; CG6808-HA (CRE): Flies after CRE/ loxP recombination; M: Molecular-weight size marker in the form of DNA ladder; wt: Wild-type flies used as a control of non-edited CG6808. (E) Results of quantitative PCR aimed to confirm the presence of the edited CG6808 gene in the lines obtained after I-Scel restriction. interest in or financial conflict with the subject matter or materials discussed in the manuscript apart from those disclosed.

No writing assistance was utilized in the production of this manuscript. 


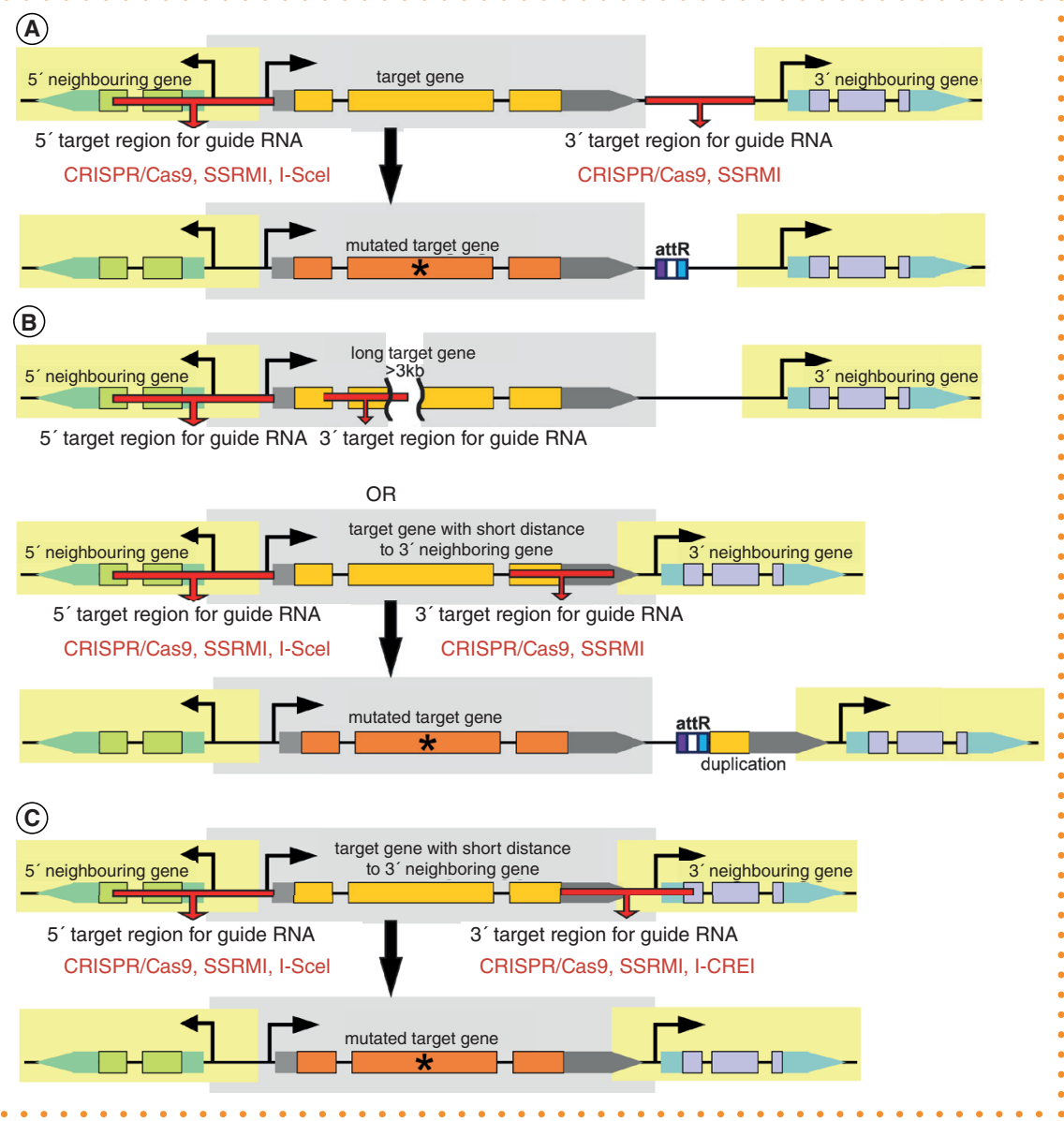

Figure 3. Summarized scheme of precise editing of the 5 '-regulatory region of the target gene. A guide RNA at the $5^{\prime}$ region of a target gene might be selected in an extended genomic region, limited only on one side by the coding region of a gene of interest. Depending on the organization of the $3^{\prime}$ region of a target gene, one of several strategies can be chosen. (A) The neighboring gene on the $3^{\prime}$ side is located at a large distance. In this case, a $3^{\prime}$ guide RNA might be chosen in the downstream region from the coding region. It is expected that an additional attR does not affect the expression of the target or neighboring gene. (B) When the target gene is large, or the neighboring gene on the $3^{\prime}$ side is in close proximity, it is possible to leave behind duplication of the $3^{\prime}$ part of the target gene. Usually, the $3^{\prime}$ part of a gene does not contain regulatory elements, whose duplication could affect gene expression. (C) In specific cases, it would be possible to use additional endonuclease, like I-CREI, that enables seamless editing at the $3^{\prime}$ region.

\section{OPEN ACCESS}

This work is licensed under the AttributionNonCommercial-NoDerivatives 4.0 Unported License. To view a copy of this license, visit http://creativecommons.org/licenses/ by-nc-nd/4.0/

\section{REFERENCES}

1. Gasiunas G, Barrangou R, Horvath P, Siksnys V. Cas9crRNA ribonucleoprotein complex mediates specific DNA cleavage for adaptive immunity in bacteria. Proc Natl Acad. Sci. USA 109(39), E2579-E2586 (2012).

2. Jinek $M$, Chylinski K, Fonfara I, Hauer M, Doudna JA Charpentier $\mathrm{E}$. A programmable dual-RNA-guided DNA
endonuclease in adaptive bacterial immunity. Science 337(6096), 816-821 (2012)

3. Gratz SJ, Ukken FP, Rubinstein CD et al. Highly specific and efficient CRISPR/Cas9-catalyzed homology-directed repair in Drosophila. Genetics 196(4), 961-971 (2014).

4. Ren X, Sun J, Housden BE et al. Optimized gene editing technology for Drosophila melanogaster using germ line-specific Cas9. Proc. Natl Acad. Sci. USA 110(47), 19012-19017 (2013)

5. Venken KJ, Schulze KL, Haelterman NA et al. MiMIC: a highly versatile transposon insertion resource for engineering Drosophila melanogaster genes. Nat. Methods 8(9), 737-743 (2011).

6. Zhang X, Koolhaas WH, Schnorrer F. A versatile twostep CRISPR- and RMCE-based strategy for efficient genome engineering in Drosophila. G3 (Bethesda) 4(12), 2409-2418 (2014)

7. Zolotarev N, Maksimenko O, Kyrchanova 0 et al. Opbp is a new architectural/insulator protein required for ribosomal gene expression. Nucleic Acids Res. 45(21) 12285-12300 (2017).

8. Yang L, Yu J. A comparative analysis of divergently-paired genes (DPGs) among Drosophila and vertebrate genomes. BMC Evol. Biol. 9, 55 (2009).

9. Dujon B. Group I introns as mobile genetic elements: facts and mechanistic speculations - a review. Gene 82(1), 91-114 (1989).

10. Colleaux L, D'Auriol L, Galibert F, Dujon B. Recognition and cleavage site of the intron-encoded omega transposase. Proc. Natl Acad. Sci. USA 85(16), 6022-6026 (1988).

11. Bellaiche $Y$, Mogila V, Perrimon N. I-Scel endonuclease a new tool for studying DNA double-strand break repair mechanisms in Drosophila. Genetics 152(3), 1037-1044 (1999).

12. Gong WJ, Golic KG. Ends-out, or replacement, gene targeting in Drosophila. Proc. Natl Acad. Sci. USA 100(5), 2556-2561 (2003)

13. Rodin S, Georgiev P. Handling three regulatory elements in one transgene: combined use of cre-lox, FLP-FRT, and I-Scel recombination systems. BioTechniques 39(6), 871-876 (2005)

14. Bischof J, Maeda RK, Hediger M, Karch F, Basler K. An optimized transgenesis system for Drosophila using germ-line-specific phic31 integrases. Proc. Natl Acad. Sci. USA 104(9), 3312-3317 (2007)

15. Aggarwal AK, Wah DA. Novel site-specific DNA endonucleases. Curr. Opin. Struc. Biol. 8(1), 19-25 (1998).

16. Arnould $S$, Chames $P$, Perez $C$ et al. Engineering of large numbers of highly specific homing endonucleases that induce recombination on novel DNA targets. J. Mol. Biol. 355(3), 443-458 (2006). 